\title{
Effect of angiotensin converting enzyme inhibitor and angiotensin II receptor blocker on the patients with sepsis
}

\author{
Hyun Woo Lee ${ }^{1,{ }^{*} \dagger}$, Jae Kyung Suh ${ }^{2,},{ }^{,}$Eunjin Jang ${ }^{3}$, and Sang-Min Lee ${ }^{1}$
}

${ }^{1}$ Division of Pulmonary and Critical Care Medicine, Department of Internal Medicine, Seoul National University Hospital, Seoul; ${ }^{2}$ National Evidence-Based Healthcare Collaborating Agency, Seoul; ${ }^{3}$ Department of Information Statistics, Andong National University, Andong, Korea

Received: August 8, 2019

Revised : October 19, 2019

Accepted: November 25, 2019

\section{Correspondence to}

Sang-Min Lee, Ph.D.

Division of Pulmonary and Critical Care Medicine, Department of Internal Medicine, Seoul National University Hospital, 101 Daehak-ro, Jongno-gu, Seoul 03080, Korea

Tel: +82-2-2072-0833

Fax: +82-2-764-2199

E-mail: sangmin2@snu.ac.kr

https://orcid.org/0000-0002-

1388-9318

*These authors contributed equally to this work.

${ }^{\dagger}$ Current affiliation: Division of Pulmonary and

Critical Care Medicine, Department of Internal Medicine, Seoul Metropolitan Government Seoul National University Boramae Medical Center, Seoul, Korea

Background/Aims: Inhibitors of the renin-angiotensin system, angiotensin-converting enzyme (ACE) inhibitors and angiotensin II receptor blockers (ARBs), reportedly have anti-inflammatory effects. This study assessed the association of prior use of ACE inhibitors and ARBs with sepsis-related clinical outcomes.

Methods: A population-based observational study was conducted using the Health Insurance Review and Assessment Service claims data. Among the adult patients hospitalized with new onset of sepsis in 2012, patients who took ARBs or ACE inhibitors at least 30 days prior to hospitalization were analyzed. Generalized linear models and logistic regression were used to examine the relation between the prior use of medication and clinical outcomes, such as in-hospital mortality, mechanical ventilation, and length of stay.

Results: Of a total of 27,628 patients who were hospitalized for sepsis, the ACE inhibitor, ARB, and non-user groups included 1,214 (4.4\%), 3,951 (14.4\%), and 22,463 (82.1\%) patients, respectively. As the patients in the ACE inhibitor and ARB groups had several comorbid conditions, higher rates of intensive care unit admission, hemodialysis, and mechanical ventilation were observed. However, after covariate adjustment, the use of ACE inhibitor (odds ratio [OR], 0.752; 95\% confidence interval [CI], 0.661 to 0.855$)$ or ARB (OR, $0.575 ; 95 \%$ CI, 0.532 to 0.621 ) was significantly associated with a lower rate of in-hospital mortality.

Conclusions: Pre-hospitalization use of ACE inhibitors or ARBs for sepsis was an independent factor for a lower rate of in-hospital mortality.

Keywords: Angiotensin-converting enzyme inhibitors; Angiotensin II; Angiotensin receptor antagonists; Mortality; Sepsis

\section{INTRODUCTION}

The renin-angiotensin system (RAS) is a complex endocrine system with a multi-dimensional enzymatic cascade to keep arterial blood pressure constant, which in turn helps maintain tissue perfusion and extracellular vol- ume [1]. The RAS is known to be physiologically activated in rodents [1] and humans [2] during sepsis. Deficiency in the expression of angiotensin-converting enzyme (ACE) and angiotensin II is associated with high mortality rates in patients with severe sepsis [3]. Experimental studies have demonstrated the 
effectiveness of angiotensin II in restoring blood pressure and cardiac output in sheep [4] and pigs [5] during septic shock. In a recent randomized controlled trial for patients with vasodilatory shock, of which approximately $80 \%$ cases were due to sepsis, administration of angiotensin II elevated blood pressure in patients resistant to conventional catecholamines as well as permitted dose reduction of other vasopressors [6].

Although RAS activation is a physiological reaction counteracting septic shock, excessive activation may further aggravate pro-inflammatory responses and vascular dysfunction, resulting in worse clinical outcomes. In particular, a high degree of RAS activation was associated with micro-vascular dysfunction and organ failure in patients with sepsis [2]. Conversely, inactivation of RAS showed protective effects on acute pulmonary or renal injury $[7,8]$ and decreased endotoxin-induced oxidative stress and endothelial dysfunction [9]. In animal models, the inhibition of angiotensin II improved mortality due to sepsis [10]. Accordingly, chronic suppression of RAS prior to an event of sepsis may be beneficial for clinical outcomes. In fact, one human observational study reported that the 30-day mortality rate due to sepsis was reduced in elderly male patients who ever received angiotensin II receptor blockers (ARBs) [11]. However, there is a lack of evidence evaluating the relationship between RAS suppression prior to an event of sepsis, and clinical prognoses.

ACE inhibitors or ARBs have been used to suppress RAS in patients with hypertension [12], cardiovascular diseases [13], and renal diseases [14]. ACE inhibitors interrupt ACE which catalyzes cleavage of angiotensin I to angiotensin II, while ARBs inhibit angiotensin II from binding to the angiotensin II receptor type I. There have been several studies to evaluate whether ACE inhibitors or ARBs can be beneficial in sepsis, but contradictory results have been reported [15-18].

The present study was conducted to determine the impact of prior RAS inhibition on the clinical prognosis during sepsis. The purpose of this study was to elucidate the effects of prior ACE inhibitor or ARB use on the in-hospital mortality rate in patients who were diagnosed with new onset of sepsis.

\section{METHODS}

\section{Study design, setting, and data sources}

We referred to The Strengthening the Reporting of Observational Studies in Epidemiology (STROBE) statement to report the results of our study [19]. A retrospective, population-based cohort study was conducted using the Health Insurance Review and Assessment Service (HIRA) annual claims data of the total population in South Korea from 2009 to 2013 . The HIRA database consists of six domains: (1) general information, (2) healthcare services, (3) diagnosis, (4) outpatient prescription, (5) drug master, and (6) providers information. Detailed information concerning HIRA data was published previously [20]. Sepsis patients who were hospitalized in a tertiary care center or general hospital in 2012 were identified using the International Classification of Diseases 1oth Revision (ICD-10) codes (A40 and A41). Considering that the definition of sepsis was revised in 2003 [21] and 2016 [22], the most recent delineation was applied to all patients in this study. No major epidemiological event or considerable change to nationwide policies was reported during this study period.

\section{Participants}

We identified all patients aged $\geq 18$ years who experienced a new event of sepsis from January 01, 2012 to December 31, 2012. Patients with recent use of ACE inhibitors or ARBs before a new event of sepsis were operationally defined as the ACE inhibitor or ARB groups, respectively. Patients who did not use ACE inhibitors or ARBs were operationally defined as the non-user group. Recent use of ACE inhibitors or ARBs was defined as a prescription given at least 30 days or more prior to the onset of sepsis. Medications used by the ACE inhibitor group included alacepril, captopril, cilazapril, enalapril, fosinopril, imidapril, lisinopril, moexipril, perindopril, ramipril, and temocapril. Medications used by the ARB group included candesartan, eprosartan, fimasartan, irbesartan, losartan, olmesartan, telmisartan, and valsartan.

\section{Measurement of variables}

We evaluated demographic characteristics such as age and sex, baseline comorbidities, Charlson comorbidity index (CCI), and current medications of hospitalized sepsis patients. Baseline comorbidities included hy- 
pertension, diabetes mellitus, cerebrovascular disorder (CVD), chronic obstructive pulmonary disorder (COPD), congestive heart failure (CHF), coronary artery disease (CAD), liver cirrhosis, chronic kidney disease (CKD), pulmonary tuberculosis, and malignancy. Comorbidities were identified by reviewing the diagnosis code (ICD10 code) up to 3 years before sepsis occurrence. Current medications other than ACE inhibitors and ARBs used within 30 days prior to the onset of sepsis, such as statins and beta-blockers, were identified. Clinical outcomes included the following events: intensive care unit (ICU) admission, mechanical ventilation, acute renal failure with hemodialysis, duration of mechanical ventilation, in-hospital mortality, hospital length of stay (LOS), and re-admission for sepsis. The event of re-admission for sepsis was followed-up until 31st December 2013. If a patient had multiple admissions to ICUs during the study period, the first episode was regarded as the major event.

\section{Assessment of outcomes}

The primary objective of our study was to evaluate the impact of current use of ACE inhibitors or ARBs on the incidence of in-hospital mortality in patients with sepsis.

\section{Statistical methods}

For comparisons among the ACE inhibitor, ARB, and non-user groups, categorical variables were analyzed using Pearson's chi-square test and presented as a percentage. Continuous variables were evaluated using analysis of variance with post hoc analysis and presented as mean \pm standard deviation. Univariate and multivariate logistic regression analyses were conducted to elucidate whether recent medication with ACE inhibitors or ARBs was associated with in-hospital mortality.

\section{Ethics}

The Institutional Review Board Committee of Seoul National University Hospital approved the present study and waived the need for informed consent for access to the HIRA data (IRB No. E-1607-004-771).

\section{RESULTS}

\section{Demographic characteristics of the study population}

A new episode of sepsis occurred in 27,365 patients in

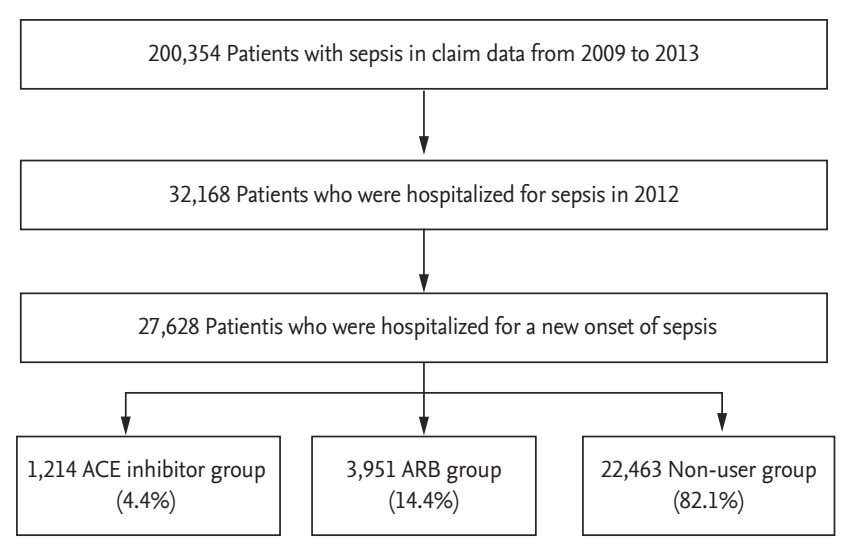

Figure 1. Flow chart of patient inclusion according to operational definitions. ACE, angiotensin-converting enzyme; $\mathrm{ARB}$, angiotensin II receptor blocker.

2012. Among them, 1,207 (4.4\%) were in the ACE inhibitor group, 3,951 (14.4\%) were in the ARB group, and $22,463(82.1 \%)$ were in the non-user group (Fig. 1). The three most commonly used drugs in the ACE inhibitor group were perindopril (34.0\%), ramipril (33.1\%), and captopril (19.7\%). The three most commonly used drugs in the ARB group were losartan (25.5\%), valsar$\tan (19.0 \%)$, and candesartan (15.0\%). The ACE inhibitor or ARB groups had a higher proportion of elderly and female patients than in the non-user group (Table 1). More patients in the ACE inhibitor or ARB group had a higher CCI than those in the non-user group. In terms of underlying conditions, more patients in the ACE inhibitor or ARB group had hypertension, diabetes mellitus, CVD, CAD, CHF, COPD, and CKD than those in the non-user group. Conversely, more patients with liver cirrhosis and malignancy were found in the non-user group. In the evaluation of current medications, a higher proportion of patients who used concurrent statins or beta-blockers was observed in the ACE inhibitor group than that in the ARB or non-user group.

In the post hoc analysis, age and sex were similar between the ACE inhibitor and ARB group (Table 2). Patients in the ARB group were more likely to have CCI $>8$ than those in the ACE inhibitor or non-user group. The ACE inhibitor group showed a higher proportion of patients with cardiac disorders such as CHF and CAD than the ARB group. The ARB group showed a higher proportion of patients with disorders related to peripheral vascular disorder such as diabetes mellitus, CVD, and 
Table 1. Baseline characteristics of hospitalized patients with sepsis, classified according to current use of ACE inhibitors, ARBs, or non-use of either drug

\begin{tabular}{|c|c|c|c|c|}
\hline Characteristic & $\begin{array}{l}\text { ACE inhibitor group } \\
(\mathrm{n}=1,207)\end{array}$ & $\begin{array}{l}\text { ARB group } \\
(\mathrm{n}=3,951)\end{array}$ & $\begin{array}{l}\text { Non-user group } \\
(\mathrm{n}=22,463)\end{array}$ & $p$ value \\
\hline Age, yr & $71.7 \pm 13.22$ & $71.04 \pm 12.25$ & $68.29 \pm 15.29$ & $<0.0001$ \\
\hline $19-40$ & $36(2.97)$ & $87(2.20)$ & $1,291(5 \cdot 75)$ & \\
\hline $41-60$ & $168(13.84)$ & $614(15 \cdot 54)$ & $4,855(21.61)$ & \\
\hline$>61$ & $1,003(82.62)$ & $3,250(82.26)$ & $16,317(72.64)$ & \\
\hline Female sex & $601(49 \cdot 51)$ & $1,977(50.04)$ & $10,445(46.50)$ & $<0.0001$ \\
\hline CCI & & & & $<0.0001$ \\
\hline $0-1$ & $126(10.38)$ & $322(8.15)$ & $3,497(15.57)$ & \\
\hline $2-8$ & $822(67.71)$ & $2,679(67.81)$ & $14,500(64.55)$ & \\
\hline$>8$ & $259(21.33)$ & $950(24.04)$ & $4,466(19.88)$ & \\
\hline \multicolumn{5}{|l|}{ Comorbidities } \\
\hline Hypertension & $349(28.75)$ & $1,109(28.07)$ & $3,466(15 \cdot 43)$ & $<0.0001$ \\
\hline Diabetes mellitus & $742(61.12)$ & $2,656(67.22)$ & $11,205(49.88)$ & $<0.0001$ \\
\hline CVD & $406(33.44)$ & $1,548(39.18)$ & $6,135(27 \cdot 31)$ & $<0.0001$ \\
\hline COPD & $278(22.90)$ & $792(20.05)$ & $3,955(17.61)$ & $<0.0001$ \\
\hline $\mathrm{CHF}$ & $440(36.24)$ & $1,136(28.75)$ & $3,970(17.67)$ & $<0.0001$ \\
\hline CAD & $486(40.03)$ & $1,403(35 \cdot 51)$ & $4,832(21.51)$ & $<0.0001$ \\
\hline Liver cirrhosis & $35(2.88)$ & $142(3 \cdot 59)$ & $1,424(6.34)$ & $<0.0001$ \\
\hline CKD & $157(12.93)$ & $690(17.46)$ & $1,612(7.18)$ & $<0.0001$ \\
\hline Pulmonary tuberculosis & $91(7.50)$ & $267(6.76)$ & $1,567(6.98)$ & 0.4197 \\
\hline Malignancy & $194(15.98)$ & $735(18.60)$ & $5,997(26.70)$ & $<0.0001$ \\
\hline \multicolumn{5}{|l|}{ Current medication } \\
\hline Statin & $385(31.71)$ & $949(24.02)$ & $1,235(5 \cdot 50)$ & $<0.0001$ \\
\hline Beta-blocker & $373(30.72)$ & $904(22.88)$ & $1,744(7 \cdot 76)$ & $<0.0001$ \\
\hline
\end{tabular}

Values are presented as mean $\pm \mathrm{SD}$ or number (\%).

ACE, angiotensin-converting enzyme; ARB, angiotensin II receptor blocker; CCI, Charlson comorbidity index; CVD, cerebrovascular disorder; COPD, chronic obstructive pulmonary disorder; CHF, congestive heart failure; CAD, coronary artery disease; CKD, chronic kidney disease.

CKD than the ACE inhibitor group. In addition, more patients in the ACE inhibitor group had COPD, while more patients in the ARB group had malignancy.

\section{Comparison of clinical outcomes according to ACE inhibitor, ARB, and non-user groups}

We found significant differences in clinical outcomes among the ACE inhibitor, ARB, and non-user groups (Table 3). A higher ICU admission rate was found in the ACE inhibitor (57.3\%) group in comparison to that in the ARB (42.7\%) and non-user (36.1\%) groups ( $p<0.0001$, both) (Table 4). Further, the ICU admission rate was higher in the ARB group than the non-user group $(p<$ $0.0001)$. Hemodialysis was more frequently conducted in the ARB group (8.5\%) than in the ACE inhibitor (5.5\%) or non-user groups $(3.1 \%)$ ( $p=0.0010$ and $p<0.0001$, respectively) and more frequently in the ACE inhibitor group than in the non-user group $(p<0.0001)$. A greater number of patients were likely to have mechanical ventilation in the ACE inhibitor (46.1\%) group than in the ARB (38.4\%) or non-user (34.5\%) groups $(p<0.0001$, both). Furthermore, more mechanical ventilation was performed for the ARB group than for the non-user group $(p<0.0001)$. The ACE inhibitor group (3.0 days) 


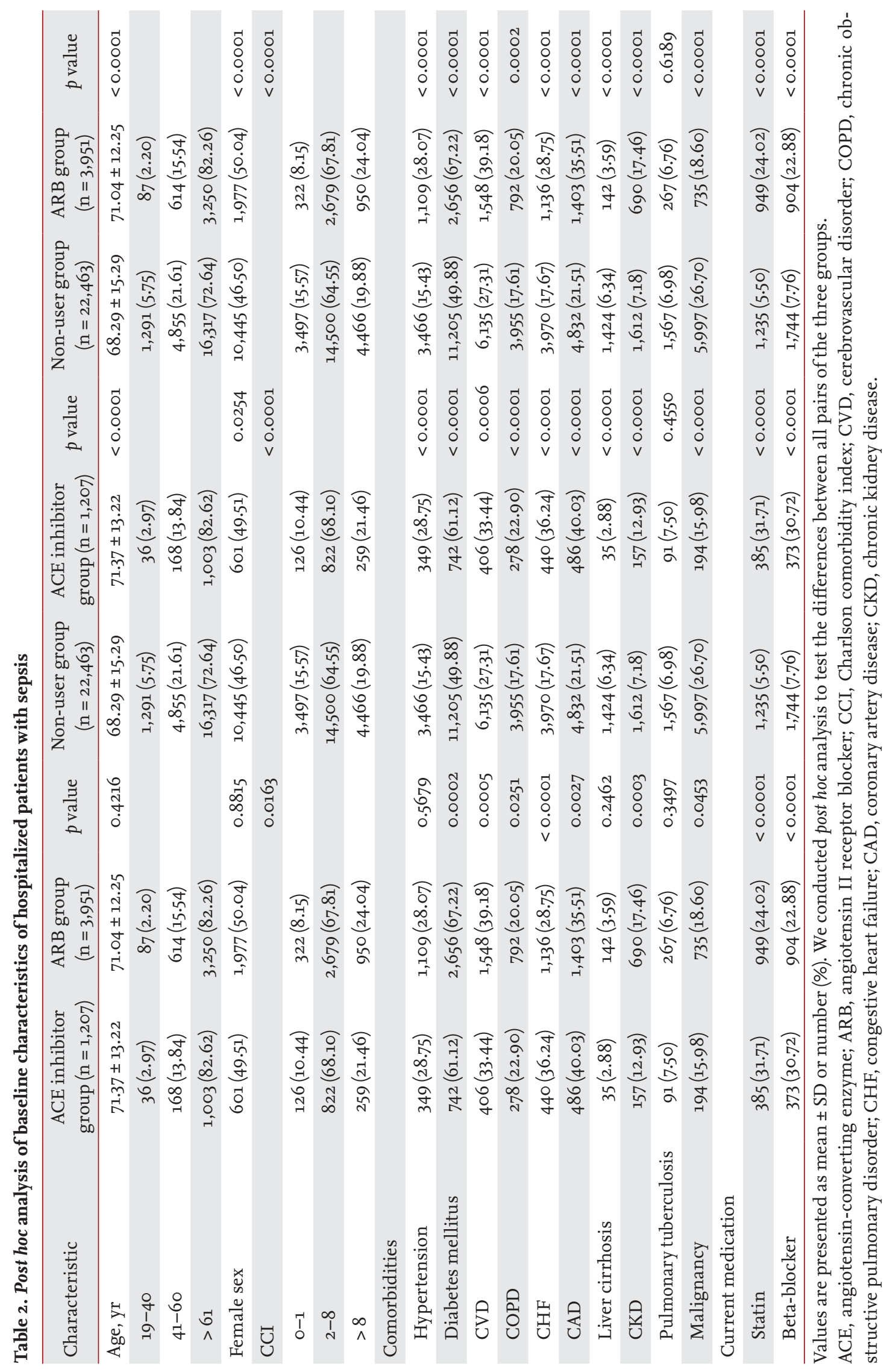


Table 3. Clinical outcomes according to the current use of ACE inhibitors and ARBs

\begin{tabular}{|c|c|c|c|c|}
\hline Variable & $\begin{array}{l}\text { ACE inhibitor group } \\
(\mathrm{n}=1,207)\end{array}$ & $\begin{array}{l}\text { ARB group } \\
(\mathrm{n}=3,951)\end{array}$ & $\begin{array}{l}\text { Non-user group } \\
\quad(\mathrm{n}=22,463)\end{array}$ & $p$ value \\
\hline Event of ICU admission & $696(57.33)$ & $1,688(42.72)$ & $8,101(36.06)$ & $<0.001$ \\
\hline Event of hemodialysis & $67(5 \cdot 52)$ & $334(8.45)$ & $699(3.11)$ & $<0.001$ \\
\hline Event of mechanical ventilation & $556(46.06)$ & $1,516(38.37)$ & $7,752(34 \cdot 51)$ & $<0.001$ \\
\hline Duration of mechanical ventilation & $2.97 \pm 1.41$ & $3.62 \pm 1.59$ & $3.74 \pm 1.58$ & 0.039 \\
\hline Hospital LOS & $41.86 \pm 39.13$ & $42.10 \pm 38.46$ & $25.04 \pm 27.92$ & $<0.001$ \\
\hline In-hospital mortality & $403(33 \cdot 39)$ & $1,174(29.71)$ & $9,376(41.74)$ & $<0.001$ \\
\hline Re-admission to ICU due to sepsis & $13(1.08)$ & $41(1.04)$ & $122(0.54)$ & $<0.001$ \\
\hline
\end{tabular}

Values are presented as or number (\%) or mean \pm SD.

ACE, angiotensin-converting enzyme; ARB, angiotensin II receptor blocker; ICU, intensive care unit; LOS, length of stay.

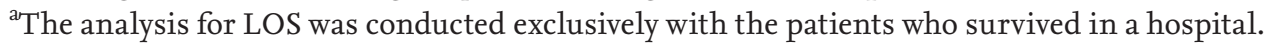

showed a significantly shorter duration of mechanical ventilation than ARB group (3.6 days) or non-user group (3.7 days) ( $p<0.0001$, both). Hospital LOS was found to be about 17 days longer in the ACE inhibitor (41.9 days) and ARB (42.1 days) groups compared to that in the non-user group (25.0 days) ( $p<0.0001$, both). During hospitalization, $29.7 \%$ patients in the ARB group, 33.4\% in the ACE inhibitor group, and $41.7 \%$ in the non-user group died. More deaths than ICU admissions were identified in the non-user group, while more ICU admissions than deaths were identified in the ACE inhibitor and ARB groups. The ARB group showed a significantly lower mortality rate than the ACE inhibitor group $(p=0.0153)$. The ICU re-admission rate due to sepsis was twice as high in the ACE inhibitor (1.08\%) and ARB (1.04\%) groups than in the non-user group $(p<0.0001$, both). No difference in ICU re-admission rates was found between the ACE inhibitor and ARB groups.

In the univariate regression analysis to evaluate the risk factors for in-hospital mortality, age, sex, CCI, comorbidities including: hypertension, diabetes mellitus, CVD, COPD, CHF, CAD, liver cirrhosis, CKD, pulmonary tuberculosis, and malignancy, ICU admission, hemodialysis, use of ACE inhibitor, use of ARB, use of statin, and use of beta-blocker were significantly related to in-hospital mortality (Table 3). After multivariable adjustment, the use of ACE inhibitor before sepsis (odds ratio [OR], 0.752; 95\% confidence interval [CI], 0.661 to 0.855 ) and ARB before sepsis (OR, 0.575; 95\% CI, 0.532 to 0.621 ) were significantly correlated to a lower mortality rate $(p<0.001$, both) (Table 5).

\section{DISCUSSION}

Our study showed that ACE inhibitor or ARB group were more likely to be elderly and have a higher number of comorbidities. Among them, CCI was higher in the ARB group. The ACE inhibitor group was associated with a greater number of underlying cardiac disorders, while the ARB group included diseases related to other vascular disorders such as cerebrovascular disease or CKD. The result that statin and beta-blockers were more often prescribed for ACE inhibitor or ARB groups also implies more underlying comorbidities in these population. Therefore, it is understandable that ICU admission, mechanical ventilation, and hemodialysis more frequently occurred in the ACE inhibitor or the ARB group. The rates of ICU admission and mechanical ventilation were the highest in the ACE inhibitor group, while the hemodialysis rate was the highest in the ARB group. The ACE inhibitor and ARB groups survived more and longer in hospital compared to non-user group. A higher mortality rate than ICU admission rate was found in the non-user group, while a higher ICU admission rate than mortality rate was found in the ACE inhibitor and ARB groups. In-hospital mortality was significantly lower in the ARB group than in the ACE inhibitor or non-user groups. Re-admission to the ICU for sepsis was more likely to occur in the ACE inhibitor and ARB groups. 


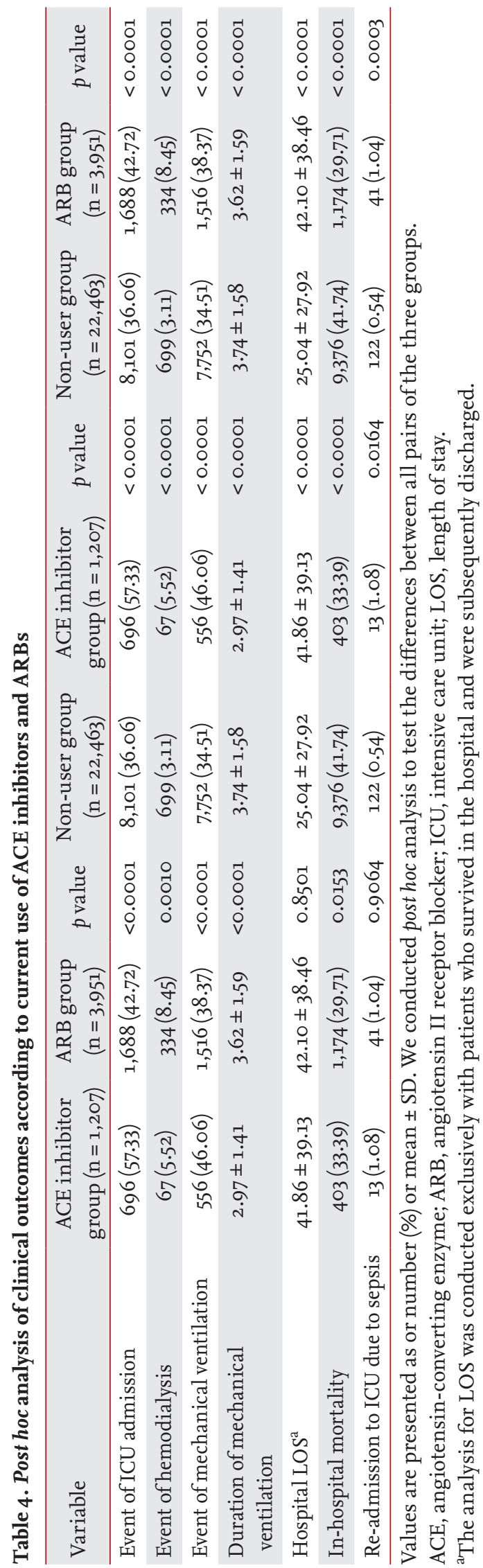

Balancing RAS activation is an important consideration for cases of sepsis. In a study by Imai et al. [7] in 2005, the protective effects of ACE-2 on lung injury was demonstrated in ACE-deficient mice. Extremely low levels of RAS expression was associated with a poor prognosis in severe sepsis [3]. In a recent clinical study, infusion of angiotensin II in septic shock patients reduced the total use of vasopressors [6]. Conversely, endotoxin-induced lung injury and inflammatory response were reduced after ACE-2 inhibition in a rat model [23]. Micro-vascular dysfunction deteriorated after RAS activation during severe sepsis [2]. An observational study showed that the use of vasopressors for septic shock decreased in patients with chronic inhibition of RAS [24]. It is well known that sepsis abruptly elevates the level of RAS expression, which induces oxidative stress, vascular permeability, pro-inflammatory cytokine production, and pro-coagulant effects $[25,26]$. Although it would be best to modulate RAS according to the severity of sepsis or the presence of shock, to date, there is no clear guideline for implementation. Based on our results, there may be more sepsis patients in whom prevention of excessive RAS activation would lead to a beneficial outcome.

The benefit of ACE inhibitors on sepsis-related clinical outcomes has been previously demonstrated. In previous systematic reviews and meta-analyses, ACE inhibitors were shown to reduce total and cardiovascular deaths in hypertensive patients [27] as well as the incidence of pneumonia and pneumonia-related mortality [28]. This reduction in mortality is largely explained by an immunomodulatory effect, which lowers inflammatory cytokines in patients with chronic heart or kidney failure [29-31]. In an experimental study, enalapril reduced systemic inflammatory response and lung injury in endotoxin-induced sepsis [32]. Our results are consistent with those of previous studies.

The immunomodulatory effect of ARB on sepsis-related outcomes has also been previously studied. Lower levels of systemic or vascular inflammation were found in patients who took ARBs [33,34]. Although previous experimental studies have revealed the relationship between angiotensin II and inflammatory processes [35], the immunomodulatory effect on patients with sepsis after blocking angiotensin II receptor is unclear. In interventional studies with rat models, losartan partially 
Table 5. Univariate and multivariate logistic regression analysis to evaluate the risk factors related with in-hospital mortality

\begin{tabular}{|c|c|c|c|c|c|c|}
\hline \multirow{2}{*}{ Variable } & \multicolumn{3}{|c|}{ Univariate analysis } & \multicolumn{3}{|c|}{ Multivariate analysis } \\
\hline & OR & $95 \%$ CI & $p$ value & OR & $95 \% \mathrm{CI}$ & $p$ value \\
\hline Age, 41-6o yr & 1.837 & $1.600-2.110$ & $<0.001$ & 1.651 & $1.433-1.902$ & $<0.001$ \\
\hline Age, $>60$ yr & 2.688 & $2.361-3.061$ & $<0.001$ & 2.532 & $2.212-2.898$ & $<0.001$ \\
\hline Female sex & 0.789 & $0.751-0.828$ & $<0.001$ & 0.819 & $0.778-0.862$ & $<0.001$ \\
\hline CCI, 2-8 & 1.317 & $1.187-1.462$ & $<0.001$ & 1.030 & $0.918-1.157$ & 0.228 \\
\hline CCI, $>8$ & 1.992 & $1.780-2.230$ & $<0.001$ & 1.319 & $1.146-1.519$ & $<0.001$ \\
\hline History of hypertension & 1.190 & $1.117-1.267$ & $<0.001$ & 1.051 & $0.979-1.128$ & 0.169 \\
\hline History of diabetes mellitus & 1.130 & $1.076-1.186$ & $<0.001$ & 0.962 & $0.91-1.018$ & 0.182 \\
\hline History of CVD & 1.145 & $1.086-1.207$ & $<0.001$ & 1.021 & $0.962-1.083$ & 0.494 \\
\hline History of COPD & 1.376 & $1.294-1.464$ & $<0.001$ & 1.098 & $1.027-1.174$ & 0.006 \\
\hline History of CHF & 1.298 & $1.222-1.378$ & $<0.001$ & 1.193 & $1.114-1.277$ & $<0.001$ \\
\hline History of CAD & 1.150 & $1.087-1.216$ & $<0.001$ & 0.976 & $0.915^{-1.041}$ & 0.457 \\
\hline History of Liver cirrhosis & 1.330 & $1.202-1.473$ & $<0.001$ & 1.170 & $1.051-1.303$ & 0.004 \\
\hline History of CKD & 1.399 & $1.286-1.521$ & $<0.001$ & 1.222 & $1.094-1.366$ & $<0.001$ \\
\hline History of Pulmonary tuberculosis & 1.568 & $1.429-1.722$ & $<0.001$ & 1.334 & $1.210-1.472$ & $<0.001$ \\
\hline History of Malignancy & 1.479 & $1.400-1.563$ & $<0.001$ & 1.274 & $1.190-1.363$ & $<0.001$ \\
\hline ICU admission for treating sepsis & 1.349 & $1.283-1.418$ & $<0.001$ & 1.437 & $1.364-1.514$ & $<0.001$ \\
\hline Hemodialysis during sepsis & 1.414 & $1.251-1.598$ & $<0.001$ & 1.198 & $1.024-1.401$ & 0.024 \\
\hline Use of ACE inhibitor before sepsis & $0.75^{1}$ & $0.665-0.849$ & $<0.001$ & $0.75^{2}$ & $0.661-0.855$ & $<0.001$ \\
\hline Use of ARB before sepsis & 0.598 & $0.556-0.643$ & $<0.001$ & 0.575 & $0.532-0.621$ & $<0.001$ \\
\hline Use of statin before sepsis & 0.684 & $0.626-0.747$ & $<0.001$ & 0.716 & $0.651-0.788$ & $<0.001$ \\
\hline Use of beta-blocker before sepsis & 0.737 & $0.680-0.799$ & $<0.001$ & 0.782 & $0.717-0.852$ & $<0.001$ \\
\hline
\end{tabular}

OR, odds ratio; CI, confidence interval; CCI, Charlson comorbidity index; CVD, cerebrovascular disorder; COPD, chronic obstructive pulmonary disorder; CHF, congestive heart failure; CAD, coronary artery disease; CKD, chronic kidney disease; ACE, angiotensin-converting enzyme; ARB, angiotensin II receptor blocker.

helped ameliorate circulation dysfunction and/or improve lung injury in septic shock [36,37]. However, only observational studies have been conducted on humans in this regard. In a population with hypertension, longterm use of ARBs showed beneficial effects in terms of the incidence of sepsis requiring hospitalization and sepsis-related outcomes [38]. Lower 30-day mortality rates were reported in elderly male patients with sepsis, who were previously prescribed with ARBs or statins [11]. A recent cohort study showed better survival outcomes in the hypertensive patients who took an anti-hypertensive drug including ACE inhibitor and ARB [39]. In this study, the adjusted analysis showed that the ARB group had a significantly lower in-hospital mortality OR than the ACE inhibitor or non-user groups.

There has been a safety concern that ACE inhibitors or
ARBs could further increase the incidence of acute kidney injury (AKI) among septic shock patients [16]. It is not a novel theory that RAS inhibition can increase AKI in critically ill patients [40]. ACE inhibitors and ARBs reduce efferent arteriolar resistance and promote renal hypoperfusion, leading to AKI [41]. Conversely, injecting angiotensin II may help reduce AKI with renal replacement therapy [42]. Therefore, ACE inhibitors or ARBs administration is usually stopped if a septic kidney is suspected, as AKI is more likely to occur in certain cases such as those with low blood pressure and intravascular hypovolemia. In our study, more events of hemodialysis due to AKI were noted in the ACE inhibitor and ARB groups. If RAS is suppressed in patients with sepsis, renal perfusion may not be enough even after hemodynamic stability is achieved. 
Several well-designed cohort studies have been recently published [39,43], but this study has some important differences. First, in this study, the method of diagnosis and treatment of sepsis were relatively uniform because we included patients over a relatively short-time period. Kim et al. [39] compared the mortality rates due to sepsis among various medications for hypertension from 2003 to 2013. The strength of the study by Kim et al. [39] is the relatively homogeneous population of hypertensive patients with long-term follow-up. In contrast, the strength of this study is the relatively homogeneous diagnostic and treatment modality because all the sepsis events were diagnosed in 2012. Second, this study extended the inclusion criteria from hypertensive patients to patients who used hypertensive medications. Hypertension was only diagnosed in approximately $30 \%$ patients who used ACE inhibitors or ARBs, while other patients used these medications for other indications. Therefore, the study population of patients with hypertension may insufficient to evaluate the effects of ACE inhibitors or ARBs on sepsis. Third, we believe that our definition of drug use was more accurate. Hsieh et al. [43] defined a patient as a user of a certain drug if the patient took that drug for $>1$ week within 3 months. Approximately $85 \%$ of all patients with sepsis were classified into the antihypertensive drug user group, and this rate was adjusted by matching the number of patients in the non-user group to the user group. Since this study only included patients who had been taking medication for at least 1 month before sepsis, the effect of taking the medication would be clearer.

Several limitations were identified in the current study. First, the present retrospective cohort study has a limitation in that clinical information was not available in the claims data. For example, the results of laboratory tests at admission or pathogen identification tests were not available. Therefore, discrepancy between actual sepsis and the disease code in the database cannot be excluded. Furthermore, clinical severity was not adjusted to evaluate clinical outcomes in this study. We could not fully explain why the ACE inhibitor or ARB groups showed better survival rates, even though these groups had more comorbidities, ICU admissions, hemodialysis treatments, and instances of mechanical ventilation. Further multicenter studies are needed to evaluate the positive effects of ACE inhibitors and ARBs on mortal- ity rates under various clinical conditions. Second, the mechanism underlying the effect of RAS suppression on the clinical course according to the severity of sepsis cannot be evaluated at this stage. Furthermore, since the effect of ACE inhibitors and ARBs on sepsis is presumed to be based on an immunomodulatory function, further analysis with various inflammatory markers is necessary. Third, the prior-exposure period of ACE inhibitors or ARBs was not the same in the study population, although we set a minimum period of 30 days. However, no previously published studies exist reporting time-dependent immunomodulatory effects of these drugs. Fourth, survival analysis could not be conducted because long-term hospitalizations occurred even after sepsis events were found. In addition, an adjusted regression analysis with the Cox proportional hazards model was not used in this study because the hazard ratios of death among the three groups were not constant over time. Fifth, the health-related activities of patients could be different depending on the patients' socioeconomic or educational status. Patients taking ACE inhibitors and ARBs are more likely to be interested in personal healthcare.

In conclusion, members of the population who were recently prescribed ACE inhibitors and ARBs at least 30 days prior to hospitalization for a new onset of sepsis had a lower rate of in-hospital mortality, although they had more comorbidities and higher rates of ICU admission, mechanical ventilation, and hemodialysis. Further clinical studies are required to determine which population segments can benefit from RAS inhibition during the early phase of sepsis.

\section{KEY MESSAGE}

1. More events of intensive care unit admission, hemodialysis, and mechanical ventilation were observed in the patients of angiotensin-converting enzyme (ACE) inhibitor and angiotensin II receptor blocker (ARB) groups who were older and had more comorbidities than those of non-user group.

2. However, the patients who used ACE inhibitor and ARB prior to hospitalization for a new onset of sepsis survived more and longer in hospital compared to non-user group. 


\section{Conflict of interest}

No potential conflict of interest relevant to this article was reported.

\section{REFERENCES}

1. Atlas SA. The renin-angiotensin aldosterone system: pathophysiological role and pharmacologic inhibition. J Manag Care Pharm 2007;13:9-20.

2. Doerschug KC, Delsing AS, Schmidt GA, Ashare A. Renin-angiotensin system activation correlates with microvascular dysfunction in a prospective cohort study of clinical sepsis. Crit Care 2010;14:R24.

3. Zhang W, Chen X, Huang L, et al. Severe sepsis: low expression of the renin-angiotensin system is associated with poor prognosis. Exp Ther Med 2014;7:1342-1348.

4. Wan L, Langenberg C, Bellomo R, May CN. Angiotensin II in experimental hyperdynamic sepsis. Crit Care 2009;13:R190.

5. Correa TD, Jeger V, Pereira AJ, Takala J, Djafarzadeh S, Jakob SM. Angiotensin II in septic shock: effects on tissue perfusion, organ function, and mitochondrial respiration in a porcine model of fecal peritonitis. Crit Care Med 2014;42:e550-e559.

6. Khanna A, English SW, Wang XS, et al. Angiotensin II for the treatment of vasodilatory shock. N Engl J Med 2017;377:419-430.

7. Imai Y, Kuba K, Rao S, et al. Angiotensin-converting enzyme 2 protects from severe acute lung failure. Nature 2005;436:112-116.

8. du Cheyron D, Lesage A, Daubin C, Ramakers M, Charbonneau P. Hyperreninemic hypoaldosteronism: a possible etiological factor of septic shock-induced acute renal failure. Intensive Care Med 2003;29:1703-1709.

9. Lund DD, Brooks RM, Faraci FM, Heistad DD. Role of angiotensin II in endothelial dysfunction induced by lipopolysaccharide in mice. Am J Physiol Heart Circ Physiol 2007;293:H3726- $\mathrm{H}_{3731 .}$

10. Laesser M, Oi Y, Ewert S, Fandriks L, Aneman A. The angiotensin II receptor blocker candesartan improves survival and mesenteric perfusion in an acute porcine endotoxin model. Acta Anaesthesiol Scand 2004;48:198204.

11. Mortensen EM, Restrepo MI, Copeland LA, et al. Impact of previous statin and angiotensin II receptor blocker use on mortality in patients hospitalized with sepsis. Pharmacotherapy 2007;27:1619-1626.

12. Ohtsubo T, Shibata R, Kai H, et al. Angiotensin-converting enzyme inhibitors versus angiotensin receptor blockers in hypertensive patients with myocardial infarction or heart failure: a systematic review and meta-analysis. Hypertens Res 2019;42:641-649.

13. Tai C, Gan T, Zou L, et al. Effect of angiotensin-converting enzyme inhibitors and angiotensin II receptor blockers on cardiovascular events in patients with heart failure: a meta-analysis of randomized controlled trials. BMC Cardiovasc Disord 2017;17:257.

14. Wang K, Hu J, Luo T, et al. Effects of Angiotensin-converting enzyme inhibitors and angiotensin II receptor blockers on all-cause mortality and renal outcomes in patients with diabetes and albuminuria: a systematic review and meta-analysis. Kidney Blood Press Res 2018;43:768779 .

15. Mortensen E, Pugh MJ, Cornell J, Anzueto A. Association of outpatient statin, ARB, and ACE inhibitor use on clinical outcomes for elderly subjects hospitalized with severe sepsis. Eur Respir J 2013;42(Suppl 57):4840.

16. Suberviola B, Rodrigo E, Gonzalez-Castro A, Serrano M, Heras M, Castellanos-Ortega A. Association between exposure to angiotensin-converting enzyme inhibitors and angiotensin receptor blockers prior to septic shock and acute kidney injury. Med Intensiva 2017;41:21-27.

17. Mawri S, Alsafadi Y, Jain T. Pre-hospital use of angiotensin-converting enzyme inhibitors and angiotensin receptor blockers is associated with improved outcomes in patients hospitalized with septic shock. Integr Clin Cardiol 2017;6:006.

18. DeMott JM, Patel G, Lat I. Effects of chronic antihypertensives on vasopressor dosing in septic shock. Ann Pharmacother 2018;52:40-47.

19. von Elm E, Altman DG, Egger M, et al. The Strengthening the Reporting of Observational Studies in Epidemiology (STROBE) statement: guidelines for reporting observational studies. Lancet 2007;370:1453-1457.

20. Kim JA, Yoon S, Kim LY, Kim DS. Towards actualizing the value potential of Korea Health Insurance Review and Assessment (HIRA) data as a resource for health research: strengths, limitations, applications, and strategies for optimal use of HIRA Data. J Korean Med Sci 2017;32:718-728.

21. Levy MM, Fink MP, Marshall JC, et al. 2001 SCCM/ESICM/ACCP/ATS/SIS International Sepsis Definitions 
Conference. Crit Care Med 2003;31:1250-1256.

22. Singer M, Deutschman CS, Seymour CW, et al. The third International Consensus Definitions for Sepsis and Septic Shock (Sepsis-3). JAMA 2016;315:801-810.

23. Li Y, Zeng Z, Li Y, et al. Angiotensin-converting enzyme inhibition attenuates lipopolysaccharide-induced lung injury by regulating the balance between angiotensin-converting enzyme and angiotensin-converting enzyme 2 and inhibiting mitogen-activated protein kinase activation. Shock 2015;43:395-404.

24. Erwin BL, Denaburg MA, Barker AB, McArdle PJ, Windham ST, Morgan CJ. Evaluation of vasopressin for septic shock in patients on chronic renin-angiotensin-aldosterone system inhibitors. Crit Care Med 2017;45:e1226-e1232.

25. Correa TD, Takala J, Jakob SM. Angiotensin II in septic shock. Crit Care 2015;19:98.

26. Benigni A, Cassis P, Remuzzi G. Angiotensin II revisited: new roles in inflammation, immunology and aging. EMBO Mol Med 2010;2:247-257.

27. Salvador GL, Marmentini VM, Cosmo WR, Junior EL. Angiotensin-converting enzyme inhibitors reduce mortality compared to angiotensin receptor blockers: systematic review and meta-analysis. Eur J Prev Cardiol 2017;24:19141924.

28. Caldeira D, Alarcao J, Vaz-Carneiro A, Costa J. Risk of pneumonia associated with use of angiotensin converting enzyme inhibitors and angiotensin receptor blockers: systematic review and meta-analysis. BMJ 2012;345:e4260.

29. Gullestad L, Aukrust P, Ueland T, et al. Effect of high- versus low-dose angiotensin converting enzyme inhibition on cytokine levels in chronic heart failure. J Am Coll Cardiol 1999;34:2061-2067.

30. Peeters AC, Netea MG, Kullberg BJ, Thien T, van der Meer JW. The effect of renin-angiotensin system inhibitors on pro- and anti-inflammatory cytokine production. Immunology 1998;94:376-379.

31. Stenvinkel P, Andersson P, Wang T, et al. Do ACE-inhibitors suppress tumour necrosis factor-alpha production in advanced chronic renal failure? J Intern Med 1999;246:503-507.

32. Hagiwara S, Iwasaka H, Matumoto S, Hidaka S, Noguchi T. Effects of an angiotensin-converting enzyme inhibitor on the inflammatory response in in vivo and in vitro mod- els. Crit Care Med 2009;37:626-633.

33. Manabe S, Okura T, Watanabe S, Fukuoka T, Higaki J. Effects of angiotensin II receptor blockade with valsartan on pro-inflammatory cytokines in patients with essential hypertension. J Cardiovasc Pharmacol 2005;46:735-739.

34. Fliser D, Buchholz K, Haller H; EUropean Trial on Olmesartan and Pravastatin in Inflammation and Atherosclerosis (EUTOPIA) Investigators. Antiinflammatory effects of angiotensin II subtype 1 receptor blockade in hypertensive patients with microinflammation. Circulation 2004;110:1103-1107.

35. Marchesi C, Paradis P, Schiffrin EL. Role of the renin-angiotensin system in vascular inflammation. Trends Pharmacol Sci 2008;29:367-374.

36. Guo J, Guo W, Jin X, Liu Y, Zhang L, Zhang J. Effects of angiotensin II type 1 receptor antagonist on rats with septic shock. Int J Clin Exp Med 2015;8:7867-7871.

37. Hagiwara S, Iwasaka H, Hidaka S, Hasegawa A, Koga H, Noguchi T. Antagonist of the type-1 ANG II receptor prevents against LPS-induced septic shock in rats. Intensive Care Med 2009;35:1471-1478.

38. Dial S, Nessim SJ, Kezouh A, Benisty J, Suissa S. Antihypertensive agents acting on the renin-angiotensin system and the risk of sepsis. Br J Clin Pharmacol 2014;78:11511158.

39. Kim J, Kim YA, Hwangbo B, et al. Effect of antihypertensive medications on sepsis-related outcomes: a population-based cohort study. Crit Care Med 2019;47:e386-e393.

40. Lim HJ, Lee HH, Kim AJ, et al. Renin-angiotensin-aldosterone system blockade in critically ill patients is associated with increased risk for acute kidney injury. Tohoku J Exp Med 2016;238:17-23.

41. Harty J. Prevention and management of acute kidney injury. Ulster Med J 2014;83:149-157.

42. Tumlin JA, Murugan R, Deane AM, et al. Outcomes in patients with vasodilatory shock and renal replacement therapy treated with intravenous angiotensin II. Crit Care Med 2018;46:949-957.

43. Hsieh MS, How CK, Hsieh VC, Chen PC. Preadmission antihypertensive drug use and sepsis outcome: impact of angiotensin-converting enzyme inhibitors (ACEIs) and angiotensin receptor blockers (ARBs). Shock 2020;53:407415 . 